Turkish Journal of Geriatrics

DOI: $10.31086 /$ tjgeri.2021.203

2021; 24(1): $87-94$

- Cengiz BURAK ${ }^{1}$

- Muhammed SÜLEYMANOĞLU1

- Ayça GÜMÜŞDAĞ ${ }^{1}$

- Murat ÇAP²

- ibrahim YILDIZ ${ }^{3}$

- Doğan İLiş1

n Şerif HAMIDEYIN ${ }^{1}$

- Fatma KÖKSAL ${ }^{4}$

- Ibrahim RENCÜZOĞULLARI ${ }^{1}$

- Yavuz KARABAĞ ${ }^{1}$

CORRESPONDANCE

${ }^{1}$ Cengiz BURAK

Kafkas University, Cardiology Clinic, Kars, Turkey

Phone: +904742251150

e-mail: cengizburaktyih@yahoo.com.tr

Received: Dec 10, 2020

Accepted: Mar 09, 2021

${ }^{1}$ Kafkas University, Cardiology Clinic, Kars, Turkey

${ }^{2}$ Gazi Yaşargil Training and Research Hospital, Cardiology Clinic, Diyarbakrr, Turkey

${ }^{3}$ Osmaniye State Hospital, Cardiology Clinic, Osmaniye, Turkey

${ }^{4}$ Mersin Training and Research Hospital, Cardiology Clinic, Mersin, Turkey

\section{THE EFFECT OF NUTRITIONAL STATUS ON LONG-TERM MORTALITY IN VERY ELDERLY PATIENTS WITH ST SEGMENT ELEVATION MYOCARDIAL INFARCTION}

\begin{abstract}
Introduction: This study aimed to evaluate the predictive role of the Geriatric Nutrition Risk Index in long-term mortality of very elderly patients with ST-segment elevation myocardial infarction and to compare it with the other known objective nutritional indices, namely the Prognostic Nutritional Index and Controlling Nutritional Status.
\end{abstract}

Materials and Methods: A total of 212 eligible patients, aged 80 years or older, who were hospitalized with a diagnosis of ST-segment elevation myocardial infarction and underwent primary percutaneous coronary intervention were included in the study. Baseline patient characteristics, echocardiographic assessments, laboratory findings, and nutritional indices were assessed.

Results: During the median follow-up period of 34 months, 60 (28.3\%) allcause mortalities were identified, and event-free cumulative rates were $46.3 \%$, $81.4 \%$, and $90.1 \%$ for Geriatric Nutrition Risk Index $<100.5,100.5-112.6$, and $>112.6$, respectively (log-rank test, $p<0.0001$ ). Receiver operating characteristic curve comparison analysis revealed that the Geriatric Nutrition Risk Index was a better predictor than the Controlling Nutritional Status, Prognostic Nutritional Index, Body Mass Index, and serum albumin ( $p<0.001$ for each pairwise comparison of Receiver operating characteristic curves).

Conclusion: It is important to evaluate malnutrition that is known to be associated with mortality in very elderly patients with ST-segment elevation myocardial infarction, who are more fragile than young people, and Geriatric Nutrition Risk Index -a simple and easy-to-calculate index- can be a guide in this regard.

Keywords: Nutritional Status; Mortality; Myocardial Infarction; Aged 


\section{INTRODUCTION}

ST-segment elevation myocardial infarction (STEMI) is one of the leading causes of death worldwide, with in-hospital mortality ranging between $4 \%$ and $12 \%$ and 1 -year mortality $10 \%(1,2)$. It has been shown that the mortality rate of elderly patients with STEMI is significantly higher than that of young patients because of their comorbidities and delayed diagnosis of myocardial infarction(3, 4). In addition, a higher proportion of elderly patients is anticipated to present with STEMI because of aging, so special consideration should be given to the risk assessment and care of these patients.

Malnutrition is a common problem affecting the elderly population and has been shown to be associated with worse clinical outcomes in patients with cancer, heart failure, and stable coronary artery disease (CAD)(5-7). To evaluate nutritional status, some indices have been developed, including the Prognostic Nutritional Index (PNI), Controlling Nutritional Status (CONUT), and Geriatric Nutrition Risk Index (GNRI)(8-10). Of these, CONUT and PNI have been shown to be related to prognosis in patients with acute coronary syndrome $(A C S)(11,12)$. Although GNRI has been validated by previous studies to assess the nutritional status of patients and predict adverse outcomes, there are limited data available in the literature on the predictive value of GNRI in patients with ACS and its comparison with other $\operatorname{scores}(7,13)$.

This study aimed to evaluate the predictive role of GNRI in long-term mortality of very elderly patients with STEMI and to compare it with the other known objective nutritional indices, namely PNI and CONUT.

\section{MATERIALS AND METHODS}

\section{Study population}

This study was conducted retrospectively by analyzing 293 patients, aged 80 years or older, who were hospitalized with a diagnosis of STEMI and underwent primary percutaneous coronary intervention (PCI) between January 2016 and December 2018 in the cardiology departments. Exclusion criteria included active inflammatory and neoplastic disease (8), end-stage renal and liver disease (6), failed primary $\mathrm{PCl}(7)$, and pretreatment with fibrinolytic drugs (2). A total of 58 patients who had missing laboratory or file data were excluded, and 212 patients were eligible for the analysis. In-hospital follow-up data were obtained from hospital file records, and post-discharge follow-up data were obtained by contacting the patients or their relatives. For patients who could not be reached, information was obtained from the National Statistical Institute and Birth Records Registry to determine if they were dead. The study protocol was reviewed and approved by the Ethics Committee of University in accordance with the Declaration of Helsinki.

\section{Data collection and definitions}

The patients' baseline clinical data, demographic data, and detailed biochemical markers-measured within 24 hours of admission-were recorded from patient files. Their height and weight were obtained from patient care forms. Detailed echocardiographic assessments were executed 24-48 hours after primary $\mathrm{PCl}$, and the left ventricular ejection fraction (LVEF) values were calculated by the modified Simpson technique. The estimated glomerular filtration rate (eGFR) was calculated from the serum creatinine levels taken on admission by the Modification of Diet in Renal Disease Study equation.

\section{Definition and calculation of nutritional scores}

Body Mass Index (BMI) was obtained for each patient by dividing the body weight $(\mathrm{kg})$ by the square of the height $\left(\mathrm{m}^{2}\right)$. GNRI was calculated as follows: GNRI $=14.89 \times$ Serum albumin $(\mathrm{g} / \mathrm{dL})+41.7 \times$ Body weight/ideal body weight, and ideal body weight 
$=($ Height $[\mathrm{cm}]-100)-($ Height $[\mathrm{cm}]-150) / 4$ for men and (Height $[\mathrm{cm}]-100)-($ Height $[\mathrm{cm}]-150) / 2$ for women(10). Subsequently, the GNRI values were divided into tertiles. CONUT (=Serum albumin [g/ $\mathrm{dL}]+$ Total lymphocyte count [per $\mathrm{mL}]+$ Total cholesterol $[\mathrm{mg} / \mathrm{dL}])$ and $\mathrm{PNI}(=10 \times$ Serum albumin $[\mathrm{g} /$ $\mathrm{dL}]+0.005 \times$ Total lymphocyte count [per $\mathrm{mL}]$ ) were calculated as previously described $(9,14)$.

\section{Statistical analysis}

Data analysis was performed using SPSS Statistics (v.22.0; SPSS Inc., Chicago, IL, USA). Continuous variables were presented as mean \pm standard deviation, and categorical variables were presented as numbers and percentages. The KolmogorovSmirnov test was used to evaluate the distribution of continuous variables. If the variables had a normal distribution, they were presented as mean \pm standard deviation; if they did not have a normal distribution, they were presented as the median (interquartile range). Categorical variables were compared using a Chi-squared test or the Fisher exact test. The Student ttest or Mann-Whitney $U$-test were used to compare the continuous variables. A two-tailed $p$-value of $<0.05$ was considered statistically significant. Univariate and multivariate Cox proportional hazard analyses were performed to identify the predictors of mortality. The KaplanMeier method was used to obtain the association between GNRI and all-cause mortality at follow-up. A receiver operating characteristic (ROC) curve was used to determine the best cutoff value for PNRI to predict all-cause mortality. Multicollinearity between GNRI and its components (albumin and BMI) was assessed by Eigenvalue and Condition Index. Linearity was tested by interacting with the logarithmic transformation of each parameter itself. To compare the area-under-the-curve (AUC) values of nutritional scores, ROC curve comparison analysis was performed using the DeLong method.

\section{RESULTS}

Of the 212 eligible patients with STEMI who underwent primary $\mathrm{PCl}$, with the mean age $86 \pm 5$ years, $18.2 \%$ were females, and the mean GNRI was 108 \pm 14 . There was no significant difference between tertiles in terms of diabetes mellitus, hypertension, dyslipidemia, smoking status, and family history of CAD. The hemodynamic parameters and rates of patients with Killip class $>1$ on admission were also not significantly different between the groups. The patients on the lowest GNRI levels had lower baseline levels of serum albumin, total cholester$\mathrm{Ol}$, and triglycerides-as well as lower eGFR and higher C-reactive protein (CRP) levels-on admission. When the other nutritional indices-PNI and CONUT-were evaluated, they were found to be in parallel with GNRI, and they increased as GNRI increased (Table 1).

Multivariate Cox regression analyses revealed that systolic blood pressure (SBP) (hazard ratio [HR]: $0.992,95 \%$ confidence interval [Cl]: 0.985-0.999, $p$ $=0.031$ ), eGFR (HR: 0.983, 95\% Cl: 0.970-0.996, $p=$ 0.010), LVEF (HR: $0.906,95 \% \mathrm{Cl}: 0.869-0.944, p<$ 0.001 ), and GNRI (HR: $0.968,95 \% \mathrm{Cl}: 0.945-0.992$, $p=0.008)$ were related with all-cause mortality (Table 2). Albumin and BMl were not included in the Cox regression analysis because of collinearity, which was observed between BMI, albumin, and GNRI.

During the median follow-up period of 34 months (interquartile range: 11-41 months), 60 (28.3\%) all-cause mortalities were identified, of which 23 (10.8\%) were in-hospital. The Kaplan-Meier analysis was performed to evaluate cumulative survival rates (Fig. 1). During the follow-up, eventfree cumulative rates were $46.3 \%, 81.4 \%$, and $90.1 \%$ for GNRI <100.5, 100.5-112.6, and >112.6, respectively (log-rank test, $p<0.0001$ ).

ROC curve analysis revealed that the optimal cut-off value of GNRI for all-cause mortality was 99.4 , with $63.3 \%$ sensitivity and $82.2 \%$ specificity 
Table 1. The baseline characteristics and laboratory results of all patients and patients classified in accordance with tertiles of GNRI

\begin{tabular}{|c|c|c|c|c|c|c|c|c|c|}
\hline \multirow[b]{2}{*}{ Age, years } & \multicolumn{2}{|c|}{$\begin{array}{l}\text { All patients } \\
\text { (n: 212) }\end{array}$} & \multicolumn{2}{|c|}{$\begin{array}{c}\text { Patients with } \\
\text { GNRI < 100.5 } \\
\text { (n: 70) }\end{array}$} & \multicolumn{2}{|c|}{$\begin{array}{l}\text { Patients with } \\
\text { GNRI 100.5-112,6 } \\
\text { (n: 71) }\end{array}$} & \multicolumn{2}{|c|}{$\begin{array}{c}\text { Patients with } \\
\text { GNRI > 112.6 } \\
\text { (n: 71) }\end{array}$} & \multirow{2}{*}{$\begin{array}{r}\text { p value } \\
0.046\end{array}$} \\
\hline & 86 & \pm 5 & 87 & \pm 5 & 86 & \pm 4 & 85 & \pm 5 & \\
\hline Female gender, n (\%) & 96 & $(45.3)$ & 27 & $(38.6)$ & 31 & $(43.7)$ & 38 & $(53.5)$ & 0.195 \\
\hline Diabetes mellitus, $n(\%)$ & 65 & $(30.7)$ & 22 & (31.4) & 24 & (33.8) & 19 & $(26.8)$ & 0.655 \\
\hline Hypertension, n (\%) & 130 & $(61.3)$ & 44 & $(62.9)$ & 43 & $(60.6)$ & 43 & $(60.6)$ & 0.950 \\
\hline Dyslipidemia, n (\%) & 71 & (33.5) & 23 & (32.9) & 24 & (33.8) & 24 & (33.8) & 0.991 \\
\hline Smoking, $n(\%)$ & 45 & $(21.2)$ & 16 & $(22.9)$ & 16 & $(22.5)$ & 13 & (18.3) & 0.764 \\
\hline Family history of CAD, $\mathrm{n}(\%)$ & 33 & $(15.6)$ & 7 & $(10.0)$ & 13 & $(18.3)$ & 13 & $(18.3)$ & 0.295 \\
\hline Killip > 1 on admission, $n(\%)$ & 51 & $(24.1)$ & 20 & $(28.6)$ & 18 & $(25.4)$ & 13 & (18.3) & 0.348 \\
\hline Systolic blood pressure, $\mathrm{mmHg}$ & 137 & \pm 39 & 134 & \pm 42 & 137 & \pm 40 & 141 & \pm 36 & 0.551 \\
\hline Heart rate, bpm & 79 & \pm 19 & 77 & \pm 21 & 81 & \pm 18 & 78 & \pm 17 & 0.365 \\
\hline White blood cell count, $10^{3} / \mu \mathrm{L}$ & 11.6 & \pm 4.0 & 12.4 & \pm 4.6 & 10.8 & \pm 3.5 & 11.7 & \pm 3.8 & 0.072 \\
\hline Lymphocyte count, $10^{3} / \mu \mathrm{L}$ & 1.3 & $(1.0-2.0)$ & 1.2 & $(1.0-1.9)$ & 1.4 & $(1.0-1.9)$ & 1.5 & $(1.1-2.1)$ & 0.361 \\
\hline Hemoglobin, g/dL & 12.4 & \pm 1.8 & 12.0 & \pm 1.9 & 12.7 & \pm 1.8 & 12.6 & \pm 1.6 & 0.028 \\
\hline eGFR, $\mathrm{ml} / \mathrm{min}$ & 71.3 & \pm 26.6 & 64.4 & \pm 26.0 & 75.3 & \pm 27.0 & 74.0 & \pm 25.6 & 0.029 \\
\hline C-Reactive protein, $\mathrm{mg} / \mathrm{dL}$ & 13.6 & (7.9-21.2) & 16.9 & $(10.2-32.5)$ & 12.1 & $(7.1-20.0)$ & 13.3 & $(7.4-16.7)$ & $<0.001$ \\
\hline Serum albumin, $\mathrm{g} / \mathrm{dL}$ & 3.48 & \pm 0.44 & 3.22 & \pm 0.38 & 3.52 & \pm 0.35 & 3.69 & \pm 0.44 & $<0.001$ \\
\hline Total cholesterol, mg/dL & 170 & \pm 45 & 160 & \pm 44 & 172 & \pm 44 & 179 & 46 & 0.038 \\
\hline $\mathrm{LDL}, \mathrm{mg} / \mathrm{dL}$ & 110 & \pm 39 & 104 & \pm 40 & 109 & \pm 40 & 116 & \pm 38 & 0.206 \\
\hline $\mathrm{HDL}, \mathrm{mg} / \mathrm{dL}$ & 41 & \pm 14 & 38 & \pm 12 & 42 & \pm 14 & 42 & \pm 17 & 0.138 \\
\hline Triglycerides, mg/dL & 106 & \pm 61 & 90 & \pm 39 & 110 & \pm 50 & 117 & \pm 84 & 0.030 \\
\hline LVEF, \% & 45 & \pm 9 & 43 & \pm 10 & 45 & \pm 8 & 46 & \pm 8 & 0.060 \\
\hline $\mathrm{BMI}, \mathrm{kg} / \mathrm{m}^{2}$ & 29.0 & \pm 5.4 & 25.1 & \pm 2.7 & 28.9 & \pm 3.5 & 34.2 & \pm 5.5 & $<0.001$ \\
\hline PNI score & 34.8 & \pm 4.4 & 32.2 & \pm 3.8 & 35.2 & \pm 3.6 & 36.9 & \pm 4.4 & $<0.001$ \\
\hline CONUT score & 175 & \pm 45 & 164 & \pm 44 & 177 & \pm 44 & 185 & \pm 46 & 0.033 \\
\hline GNRI score & 108 & \pm 14 & 93 & \pm 6 & 107 & \pm 4 & 123 & \pm 8 & $<0.001$ \\
\hline In hospital death, n (\%) & 23 & $(10.8)$ & 13 & (18.6) & 6 & (8.5) & 4 & $(5.6)$ & 0.034 \\
\hline Long-term death, n (\%) & 37 & (19.6) & 26 & $(45.6)$ & 8 & (12.3) & 3 & (4.5) & $<0.001$ \\
\hline Total death, n (\%) & 60 & $(28.3)$ & 39 & $(55.7)$ & 14 & $(19.7)$ & 7 & (9.9) & $<0.001$ \\
\hline
\end{tabular}

Abbreviations: GNRI; Geriatric Nutritional Risk Index, CAD; coronary artery disease, eGFR; estimated glomerular filtration rate, LDL; low density lipoprotein, $\mathrm{HDL}$; high density lipoprotein, LVEF; left ventricular ejection fraction, PNI; prognostic nutritional index, CONUT; controlling nutritional status. 
(AUC: $0.791,95 \% \mathrm{Cl}: 0.708-0.859, \mathrm{p}<0.001$ ). ROC curve comparison analysis was performed to compare nutritional scores, as well as serum albumin and BMI. GNRI was found to be a better predictor than CONUT (AUC: $0.603,95 \% \mathrm{Cl}: 0.510-0.690)$, PNI (AUC: $0.618,95 \% \mathrm{Cl}: 0.526-0.704), \mathrm{BMI}$ (AUC: 0.616, 95\% Cl: 0.524-0.702), and serum albumin (AUC: 0.624, 95\% Cl: 0.532-0.710; $p<0.001$ for each pairwise comparison of ROC curves) (Fig. 2).

\section{DISCUSSION}

This study evaluated the predictive value of GNRI in long-term mortality in patients, aged 80 years or older, with STEMI and the comparison of nutritional indices among themselves. Consequently, low GNRI was an independent predictor of long-term mortality and was superior to the other nutritional indices, namely BMI, PNI, and CONUT, in this age group.

Table 2. Univariable and multivariable Cox regression analysis for the prediction of total mortality

\begin{tabular}{|l|c|c|c|c|}
\hline \multicolumn{2}{|c|}{ Univariable analysis } & \multicolumn{2}{c|}{ Multivariable analysis } \\
\hline & p value & HR (95\% Cl) & p value & HR (95\% Cl) \\
\hline Systolic blood pressure & $<0.001$ & $0.986(0.979-0.994)$ & 0.031 & $0.992(0.985-0.999)$ \\
\hline Estimated glomerular filtration rate & $<0.001$ & $0.963(0.951-0.976)$ & 0.010 & $0.983(0.970-0.996)$ \\
\hline Left ventricular ejection fraction & $<0.001$ & $0.880(0.848-0.913)$ & $<0.001$ & $0.906(0.869-0.944)$ \\
\hline GNRI & $<0.001$ & $0.928(0.906-0.952)$ & 0.008 & $0.968(0.945-0.992)$ \\
\hline
\end{tabular}

All clinically relevant parameters were included in the model. Abbreviations: GNRI; Geriatric Nutritional Risk Index

Figure 1. Kaplan Meier survival analysis of long-term mortality in patients classified in accordance with tertiles of Geriatric Nutrition Risk Index

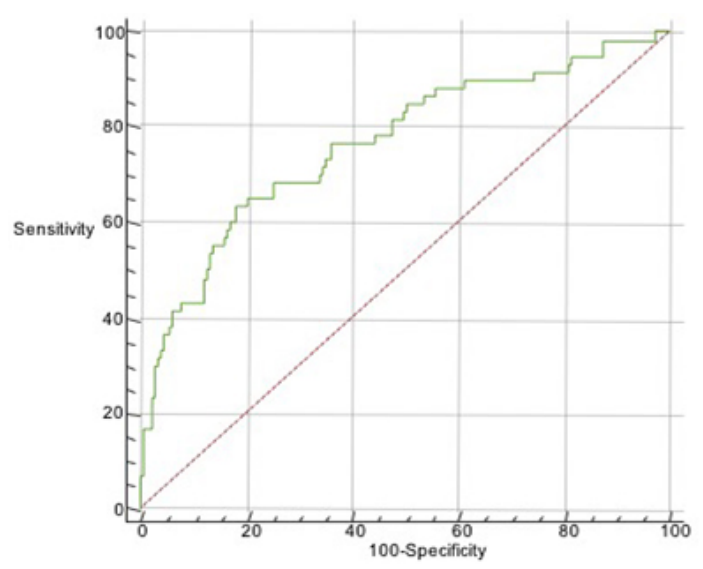

Figure 2. ROC curve comparison analysis of the nutritional scores and also serum albumin and Body Mass Index

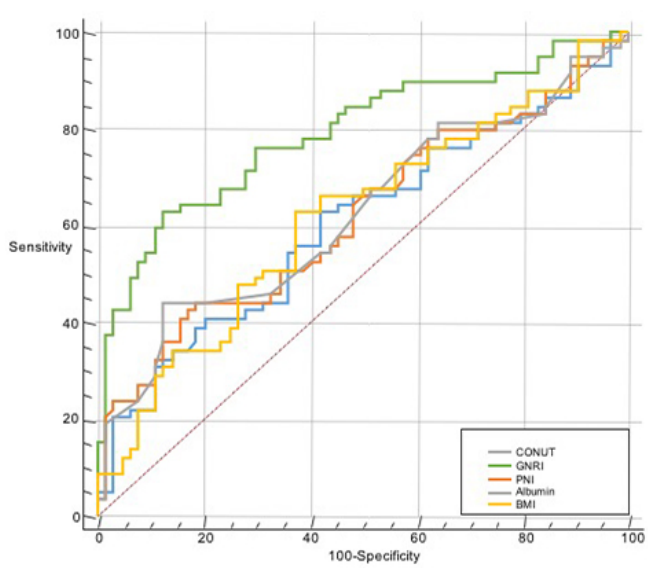


Malnutrition is one of the reasons leading to worse outcomes in the elderly population, and the aging process itself is a cause for malnutrition, so the evaluation of nutritional status in elderly patients seems important. Basically, it has been demonstrated that serum albumin and BMI can be used to assess nutritional status. Low serum albumin and BMI have been associated with worse clinical outcomes, including mortality in patients with $\operatorname{ACS}(12,15)$. In the present study, serum albumin and BMl were evaluated, and in parallel with the literature, they were significantly lower in patients with low GNRI. The relationship of low albumin and BMI with mortality may be related to the change in cardiometabolic demands and priorities in ACS. Because cardiometabolic demands are increased because of the activation of neurohormonal and inflammatory pathways in ACS, patients with low BMl who have low physiological reserves and fat stores may not be able to overcome these catabolic changes(16). Infection, heart failure, and other cardiac reasons may require hospitalization after discharge in patients with low BMI, which also leads to additional weight loss and, as a result, increases long-term mortality. Similarly, serum albumin is a widely used marker to assess nutritional status, and its level decreases as a result of malnutrition. Besides, it is a negative acutephase reactant, and its decrease in an inflammatory state such as ACS may have had an additive effect and was significantly lower in patients with malnutrition. In addition, CRP is an inflammatory marker, and its increased level has been shown to be a predictor for worse clinical outcomes in patients with ACS(17). In accordance with the literature, we found higher CRP levels in patients with low GNRI. According to this result, it can be speculated that malnutrition aggregates the inflammatory state.

In the Cox regression analysis to predict total mortality, SBP, eGFR, and LVEF were found to be independent predictors, and this result is consistent with the literature $(18,19)$. In addition, we found that GNRI was an independent predictor of mortality in very old patients with STEMI. The assessment of nutritional status involves the process of obtaining, verifying, and interpreting the data needed to discover nutritional problems and their causes and importance(20). To ensure a structured assessment and documentation of nutritional status, it is important to identify which nutritional assessment tool is appropriate for use in that group. The prognostic value of $\mathrm{PNI}$ in predicting poor prognosis, including mortality, has been tested in patients with STEMI, and PNI has been found to be an independent predictor of mortality $(11,21)$. In another study, patients with severe CONUT-not those with severe PNIhad the highest event rate for all-cause mortality in patients with STEMI(12). In these studies, GNRI has not been tested, and patient groups were younger than the patients in the present study because the mean age of patients was between 58 and 65 years in the aforementioned studies. Therefore, considering that nutritional problems increase with age, it can be said that data on the evaluation and importance of this in the population, aged 80 years and older, with STEMI are limited. In the present study, we found that GNRI predicts mortality and is superior to $\mathrm{PNI}$ and CONUT in very elderly patients.

GNRI was specifically designed to assess the nutritional status of elderly patients and predict malnutrition-related complications, and its validity and reliability have been better studied in hospitalized elderly patients than in those with STEMI(7, 10, 22-24). In the present study, we found that low GNRI was an independent predictor of mortality and was superior to $\mathrm{BMI}, \mathrm{PNI}$, and CONUT in very old patients with STEMI. Recently, the Mini-Nutritional Short Form (MNA-SF) was used to evaluate the nutritional status of elderly patients with ACS, and it was found to be a useful predictor for allcause mortalities(25). Although we did not evaluate the effectiveness of MNA-SF in the present study, it was found that GNRI was more appropriate than MNA-SF in assessing nutritional status and identifying nutritional complications in hospitalized elderly 
patients(23). Moreover, using GNRI to evaluate malnutrition is a less time-consuming and easy tool that requires fewer medical staff.

Malnutrition is associated with mortality not only in hospitalized elderly patients but also in the normal population hospitalized with ACS. Moreover, albumin is used in addition to BMI in the calculation of GNRI, and albumin is both a negative acute-phase reactant and a marker of malnutrition. Depending on all this, it may have had an additive effect on GNRI being a strong predictor of mortality in elderly patients with STEMI.

As a conclusion, the number of elderly patients with STEMI is increasing because of aging, and it

\section{REFERENCES}

1. Kristensen SD, Laut KG, Fajadet J, et al. Reperfusion therapy for ST elevation acute myocardial infarction 2010/2011: current status in 37 ESC countries. Eur Heart J. 2014;35(29):1957-70. (PMID: 24419804)

2. Pedersen $F$, Butrymovich $V$, Kelbaek $H$, et al. Shortand long-term cause of death in patients treated with primary PCl for STEMI. J Am Coll Cardiol. 2014;64(20):2101-8. (PMID: 25457398)

3. Oduncu V, Erkol A, Tanalp AC, et al. Comparison of early and late clinical outcomes in patients $>=80$ versus $<80$ years of age after successful primary angioplasty for ST segment elevation myocardial infarction. Turk Kardiyol Dern Ars. 2013;41(4):319-28. (PMID: 23760119)

4. Claessen BE, Kikkert WJ, Engstrom AE, et al. Primary percutaneous coronary intervention for ST elevation myocardial infarction in octogenarians: trends and outcomes. Heart. 2010;96(11):843-7. (PMID: 19966111)

5. Honda Y, Nagai T, Iwakami N, et al. Usefulness of Geriatric Nutritional Risk Index for Assessing Nutritional Status and Its Prognostic Impact in Patients Aged $>/=65$ Years With Acute Heart Failure. Am J Cardiol. 2016;118(4):550-5. (PMID: 27324158)

6. Zheng HL, Lu J, Li P, et al. Effects of Preoperative Malnutrition on Short- and Long-Term Outcomes of Pa- is important to evaluate these patients more meticulously and objectively. As malnutrition is a known predictor of mortality, it may be important to evaluate it with a simple, cost-effective, and easily calculated index.

\section{Acknowledgement; None to declare}

Funding; This research did not receive any specific grant from funding agencies in the public, commercial, or not-for-profit sectors.

Disclosures; The authors declare that there is no conflict of interest.

tients with Gastric Cancer: Can We Do Better? Ann Surg Oncol. 2017;24(11):3376-85. (PMID: 28699132)

7. Wada H, Dohi T, Miyauchi K, et al. Prognostic Impact of the Geriatric Nutritional Risk Index on Long-Term Outcomes in Patients Who Underwent Percutaneous Coronary Intervention. Am J Cardiol. 2017;119(11):1740-5. (PMID: 28388993)

8. Buzby GP, Mullen JL, Matthews DC, et al. Prognostic nutritional index in gastrointestinal surgery. Am J Surg. 1980;139(1):160-7. (PMID: 7350839)

9. Ignacio de Ulibarri J, Gonzalez-Madrono A, de Villar NG, et al. CONUT: a tool for controlling nutritional status. First validation in a hospital population. Nutr Hosp. 2005;20(1):38-45. (PMID: 15762418)

10. Bouillanne O, Morineau G, Dupont C, et al. Geriatric Nutritional Risk Index: a new index for evaluating at-risk elderly medical patients. Am J Clin Nutr. 2005;82(4):777-83. (PMID: 16210706)

11. Keskin M, Hayiroglu MI, Keskin T, et al. A novel and useful predictive indicator of prognosis in ST-segment elevation myocardial infarction, the prognostic nutritional index. Nutr Metab Cardiovasc Dis. 2017;27(5):438-46. (PMID: 28392077)

12. Basta G, Chatzianagnostou K, Paradossi U, et al. The prognostic impact of objective nutritional indices in elderly patients with ST-elevation myocardial infarc- 
tion undergoing primary coronary intervention. Int J Cardiol. 2016;221:987-92. (PMID: 27441479)

13. Cereda E, Limonta D, Pusani C, et al. Geriatric nutritional risk index: a possible indicator of short-term mortality in acutely hospitalized older people. J Am Geriatr Soc. 2006;54(6):1011-2. (PMID: 16776806)

14. Onodera T, Goseki N, Kosaki G. [Prognostic nutritional index in gastrointestinal surgery of malnourished cancer patients]. Nihon Geka Gakkai Zasshi. 1984;85(9):1001-5. (PMID: 6438478)

15. Sujino Y, Tanno J, Nakano S, et al. Impact of hypoalbuminemia, frailty, and body mass index on early prognosis in older patients ( $>/=85$ years) with ST-elevation myocardial infarction. J Cardiol. 2015;66(3):2638. (PMID: 25547740)

16. O'Brien EC, Fosbol EL, Peng SA, et al. Association of body mass index and long-term outcomes in older patients with non-ST-segment-elevation myocardial infarction: results from the CRUSADE Registry. Circ Cardiovasc Qual Outcomes. 2014;7(1):102-9. (PMID: 24326936)

17. Marcucci R, Valente S, Gori AM, et al. Global platelet hyperreactivity and elevated C-reactive protein levels predict long term mortality in STEMI patients. Thromb Res. 2014;134(4):884-8. (PMID: 25135796)

18. Anavekar NS, McMurray JJ, Velazquez EJ, et al. Relation between renal dysfunction and cardiovascular outcomes after myocardial infarction. N Engl J Med. 2004;351(13):1285-95. (PMID: 15385655)

19. Ng VG, Lansky AJ, Meller S, et al. The prognostic importance of left ventricular function in patients with ST-segment elevation myocardial infarction: the HORIZONS-AMI trial. Eur Heart J Acute Cardiovasc Care. 2014;3(1):67-77. (PMID: 24562805)

20. Field LB, Hand RK. Differentiating malnutrition screening and assessment: a nutrition care process perspective. J Acad Nutr Diet. 2015;115(5):824-8. (PMID: 25582410)

21. Chen QJ, Qu HJ, Li DZ, et al. Prognostic nutritional index predicts clinical outcome in patients with acute ST-segment elevation myocardial infarction undergoing primary percutaneous coronary intervention. Sci Rep. 2017;7(1):3285. (PMID: 28607442)

22. Cereda E, Pedrolli C. The Geriatric Nutritional Risk Index. Curr Opin Clin Nutr Metab Care. 2009;12(1):1-7. (PMID: 19057180)

23. Abd-El-Gawad WM, Abou-Hashem RM, El Maraghy $\mathrm{MO}$, et al. The validity of Geriatric Nutrition Risk Index: simple tool for prediction of nutritional-related complication of hospitalized elderly patients. Comparison with Mini Nutritional Assessment. Clin Nutr. 2014;33(6):1108-16. (PMID: 24418116)

24. Komatsu M, Okazaki M, Tsuchiya K, et al. Geriatric Nutritional Risk Index Is a Simple Predictor of Mortality in Chronic Hemodialysis Patients. Blood Purif. 2015;39(4):281-7. (PMID: 25925239)

25. Tonet E, Campo G, Maietti E, et al. Nutritional status and all-cause mortality in older adults with acute coronary syndrome. Clin Nutr. 2020;39(5):1572-9. (PMID: 31324416) 